\title{
Kinematics of dSph and dE galaxies
}

\author{
Mario Mateo \\ Department of Astronomy; University of Michigan; Ann Arbor, MI 48109-1042, USA
}

\begin{abstract}
We have entered a new era in the observational study of the kinematics of dwarf spheroidal and dwarf elliptical galaxies. Large telescopes with multi-object spectrographs can now obtain radial velocities of hundreds if not thousands of individual stars in the nearer dSph systems, while new sensitive longslit spectrometers can measure the kinematics of $\mathrm{dE}$ galaxies from their integrated light to unprecedented faint surface brightness levels. I review where we have been, where we are, and where we are going in the kinematic studies of these dwarfs systems.
\end{abstract}

\section{Introduction}

The attendees to IAUC 198 and possibly many of the readers of this review need little motivation to justify the study of dwarf Spheroidal (dSph) and dwarf Elliptical (dE) galaxies. These systems have proven to be closely related to the earliest dark matter (DM) halos to have ever formed: they are both high-density and small, pushing them to the earliest formation epochs in any hierarchical cosmology (Press and Schechter 1974; Navarro, Frenk and White 1997). We see direct evidence that dwarfs are merging into large, local structures (Ibata et al. 1994), and that this process may be ubiquitous at the present epoch (Shang et al. 1998; Ferguson et al. 2002). Because dwarfs live in such an extreme gravitational state - their stars reside in extremely broad, and hence, lowacceleration potentials - they are natural tests of non-standard gravity models (Gerhard and Spergel; Łokas 2001, 2002). These small galaxies can be used to address some of the biggest issues in astrophysics; their kinematics play an important role in this regard.

But it was not always this way. It is well known that Marc Aaronson almost singlehandedly started the field of $\mathrm{dSph} / \mathrm{dE}$ with his seminal 1983 paper. Though Aaronson clearly wanted to use this paper to launch investigations to the sorts of issues I summarized above, everyone else met it with far more prosaic questions. How could one claim to derive any useful information on the dynamical state of a pressure-supported system with just three (!) stars? How could one possibly use Carbon stars - some known to be variables - as kinematic tracers at the few $\mathrm{km} / \mathrm{s}$ level? And what about binary stars? These might contribute significantly to the inferred dispersions of 8-10 km/s. Many wondered how one could possibly obtain $1 \mathrm{~km} / \mathrm{s}$ precision for such very faint stars $(V \sim 18$ !).

New telescopes and instruments have rejuvenated the field of $\mathrm{dSph} / \mathrm{dE}$ kinematics, providing the opportunity to obtain large samples of stars in the nearer systems, and making it possible to carry out integrated-light spectroscopy at high enough resolution to study dE systems beyond the Local Group. My own efforts are built around a new instrument - the Michigan/MIKE Fiber system - on the Magellan telescope. With MMFS we can obtain spectra at a resolution of 15,000 to 19,000 of up to 256 targets simultaneously. We observe the region from 5120-5200 $\AA$ which contains strong Mg features and many weaker (mostly Fe) lines. In $2-3$ hours we obtain sufficient $\mathrm{S} / \mathrm{N}$ to $V \sim 21$ to measure velocities of late-type giants to a precision of $1-2 \mathrm{~km} / \mathrm{s}$. We have spectra now of over 2500 stars in six galaxies; by comparison, all of the kinematic results summarized in Mateo 1998 were based on observations of about 450 stars. 
The early concerns - sample size, stellar atmospheric and binary motions, and the intrinsic velocity precision of the observations - consumed the early discussions of dSph kinematics through much of the 1980's. They remain important issues, and it is important to not ignore these more prosaic questions. Nonetheless, as these problems become more settled, we can turn to the much more interesting questions that $\mathrm{dSph} / \mathrm{dE}$ kinematics address. Do the kinematics of these dwarfs arise solely from internal effects? If external effects matter, what are these processes and do they operate on all dSph to a similar degree? Finally, are the basic assumptions we bring to the analysis of these systems secure? To make for a more constrained discussion here, I will address four issues below that relate to these more general questions.

\section{Does mass follow light in $\mathrm{dSph} / \mathrm{dE}$ galaxies?}

Pryor and Kormendy (1990) first tried to address this question using only data on the central velocity dispersions of two dSph satellites of the Milky Way. Not surprisingly, it was impossible to rule out or confirm extended DM halos from these data; what was clear was that dispersion profiles were required. Figure 1 is a plot of dispersion profiles for five nearby systems based on observations of 1400 stars (Mateo et al. 2005, Walker et al. 2005). Dispersion profiles have also been produced for some of these galaxies by others, including Sextans (Kleyna et al. 2004), Draco (Kleyna et al. 2001), Sculptor (Tolstoy et al. 2004), and Leo I (Sohn et al. 2005). In Sculptor, the dispersion profiles appear to depend on metallicity, a potentially very exciting result (see below). Dispersion and rotation profiles are now also being produced for $\mathrm{dE}$ galaxies as distant as the Virgo and Fornax clusters (van Zee et al. 2004; Geha et al. 2002, 2003; De Rijcke et al. 2001, 2004). Apart from the very closest dEs (e.g. NGC 205, Geha 2004), this work remains limited to integrated spectroscopy of galaxies with surface brightnesses considerably higher than non-nucleated dSph galaxies. Nonetheless, the results as they stand suggest a plausible (but not yet proven) kinematic connection between dSph galaxies (at the low surface brightness, low mass, and small size end of the relations) and dE's (see Geha et al. 2003).

Among the dE galaxies, what is immediately evident is the sheer variety in the radial kinematic profiles of these galaxies. In contrast, the situation for dSph galaxies is somewhat less clear; some studies claim to see cold cores (Sextans; Kleyna et al. 2004), with other profiles falling at large radii (Ursa Minor and Draco; Wilkinson et al. 2004). The flat profiles in Figure 1 for some of the same galaxies suggest that the purported variety of the dSph kinematics may be due to small-sample effects. While none of the lowestluminosity dSph systems exhibit dynamically significant rotation, some dE galaxies some minor-axis rotation, while others - for no recognizable reason (e.g. Geha et al. 2003) show no rotation at all. For these galaxies their shapes appear to be maintained either fully by internal pressure support or fully by rotation.

But what of our question above? In every case measured where the kinematic data extend well outside the core radii of dSph and (a few) dE systems, dispersion profiles appear to strongly favor mass profiles, $M(R)$, that extend beyond the limits of the visible components of the galaxies (e.g Wang et al. 2005). There is also growing indications that the kinematics become progressively more radially anisotropic, at least in some dSph galaxies (Wilkinson et al. 2002), as stars approach the classical tidal radii. Walker et al. (2005) have specifically addressed this issue in Fornax. Under the assumptions of spherical symmetry and an isotropic velocity distribution, they find that mass must be considerably more extended than visible matter in that galaxy. Early results for the other dSph systems in Figure 1 confirm this conclusion under the same assumptions. Future 

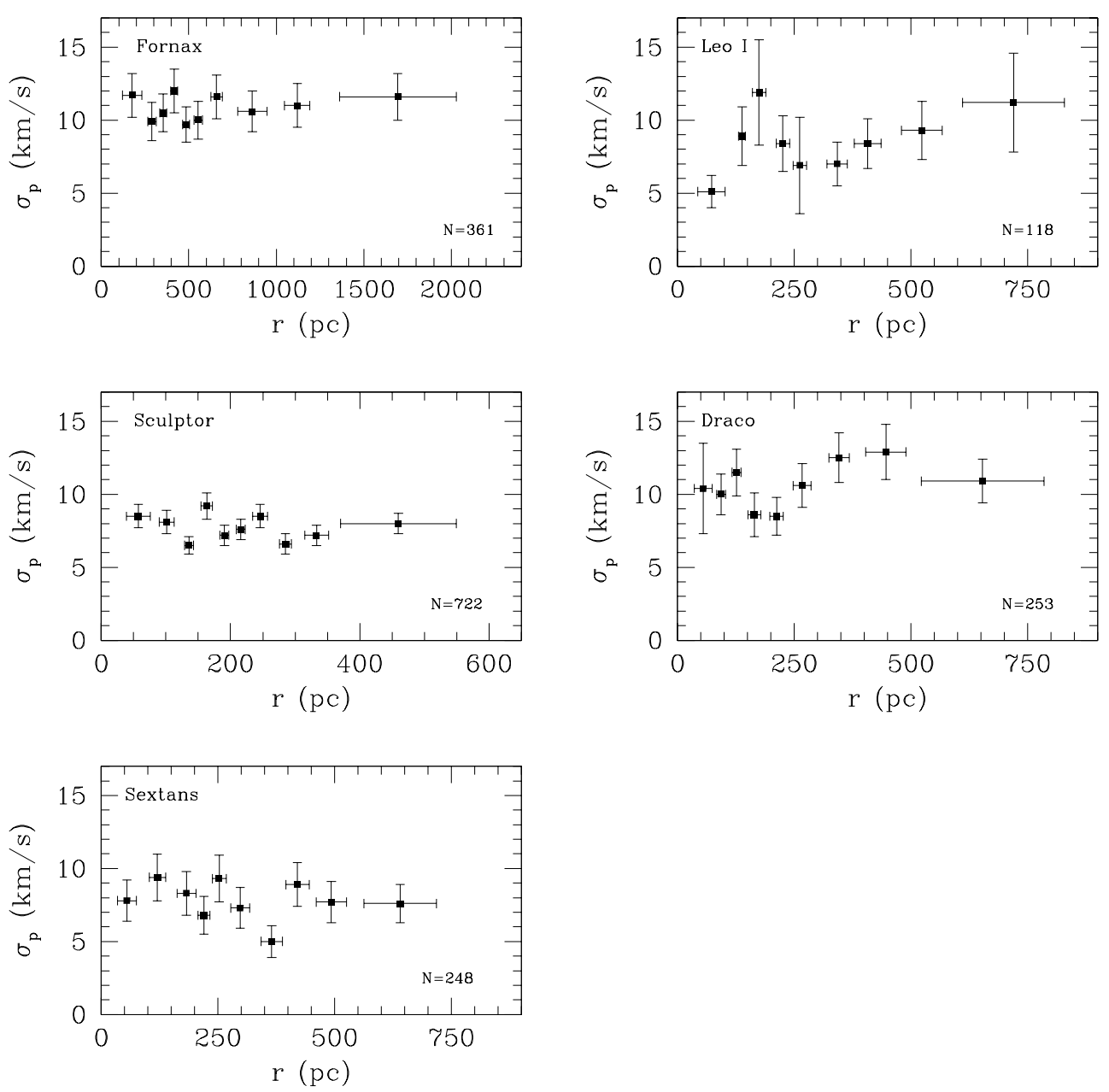

Figure 1. Binned radial velocity dispersion profiles for five of the Milky Way's dSph satellite companions. The data summarized here were taken with the Michigan/MIKE Fiber System at the Magellan Observatory (For, Scl, and Sex) or with Hectochelle at the MMT (Leo I and Dra).

work will clearly revolve around the validity of these assumptions in real systems and how to drop them when they are not justified (see Wilkinson et al. 2002).

\section{Substructure in $\mathrm{dE}$ and dSph Galaxies}

Despite the low luminosities and seemingly simple structures of dwarf ellipsoidal systems, these galaxies frequently display internal substructure. Geha et al. $(2002 ; 2003)$ produced some extraordinary maps that illustrate the nature and variety of substructures present in dE galaxies. Some show extended halos, some bright cores, some inner disks and some miniature spiral patterns. The variety is astounding, but no clear correlation of these structures with external (location relative to neighboring systems), or internal properties (luminosity, kinematics, surface brightness) has been established. 
Most dSph galaxies reveal star-formation histories that are decidedly non-monotonic (Mateo 1998), and which often exhibit complicated variations with location within individual systems. The latter is usually seen in the form of a core-halo age or color gradient (Harbeck et al. 2001; Tolstoy et al. 2004), but highly asymmetric structures such as clumps and possibly shells (Coleman et al. 2004, Coleman and Da Costa 2005) are also seen. These stellar population variations have long fueled speculation that dSph galaxies in particular may have evolved from low-surface brightness dIrr systems (Mayer et al. 2001a,b). The behavior of the core of NGC 205 is particularly instructive. Here, tidal effects seem to have driven gas towards the galaxy center where star formation occurs within a kinematically cold core (McConnachie et al. 2004; Geha 2004; Butler and Martínez-Delgado 2005). Since much of the substructure seen in other dE systems tends to focus near the galaxy cores (Geha et al. 2002, 2003), and so similar tidal influences may be the ultimate cause.

The shells described by Coleman et al. (2004. 2005) are more surprising, as they may indicate that some dSph galaxies have undergone mergers with yet smaller systems in the recent past. These authors note that the substructure in this particular galaxy has a different age distribution than the galaxy as a whole (see also Olszewski et al. 2005) and that the purported merger has produced a halo-like structure aligned with, presumably, the plane along which the merged system entered Fornax (which happens to be nearly perpendicular to the major axis of Fornax).

Kleyna et al. (2003) illustrate the use of substructure as a dynamical probe by modeling the kinematically distinct clump offset from the core of the Ursa Minor dSph galaxy. Their observations confirm the system to be a cold-substructure within the overall potential of UMi. Their models demonstrate such a structure can only survive if the central potential of UMi arises from a near-constant density central DM halo, one with an effective core radius much larger than that of the visible material. It remains to be seen how substructure in other dwarfs can be exploited to constrain the underlying potentials of these systems, but this lesson in UMi illustrates that the kinematics of substructure can be most revealing. Direct kinematic evidence of substructure has also been detected in some of the more luminous $\mathrm{dE}$ systems in the form of strong core-halo kinematic discontinuities (Geha et al. 2003; De Rijcke et al. 2001, 2004).

\section{The Role of Tides}

There is simply no question now that tides affect the structures and kinematics of dwarf galaxies. We see strong tidal signatures in systems ranging from the Magellanic Clouds to fully disrupted streams orbiting the Milky Way and nearby galaxies. The crucial question is 'to what extent to tides dominate the kinematics of dwarfs?' Much of the motivation behind the kinematic surveys of $\mathrm{dSph}$ and $\mathrm{dE}$ systems is to constrain the properties of their dark-matter halos. But if tides dominate, such analyses are at worst invalid if they rely on static models such as King or NFW profiles, and at best greatly complicated by the need to include the role of tides. As illustrated by the many high-resolution n-body simulations presented at this meeting, tides have conceivably have affected all of the dwarf satellites of our Milky Way to some extent.

Closer to the Milky Way the distinction between internal kinematics and tidal effects becomes more problematic. At this meeting Evans et al. argued that Draco shows a distinct drop in its dispersion profile at large radii, a hint that tides do not necessarily dominate that galaxy's kinematics. An independent measurement of this profile (Mateo et al. 2005; see Figure 1) does not confirm this result. In the case of Ursa Minor, another dSph plausibly affected strongly by tides, Palma et al. (2003) and Gómez-Flechoso and 
Martínez-Delgado (2003) suggests that UMi merges into a stream-like outer structure aligned with the galaxy's projected space-motion. The fact that both Draco and UMi lie close to an 'exclusion boundary' of 60-70 kpc within which dSph galaxies are rarely seen surrounding $L_{*}$ galaxies provides some empirical hint that perhaps these systems may be showing incipient tidal effects.

My goal here is not to try to settle this particular debate, but to emphasize that to better account for tides we need (a) better constraints on the form of the Milky Way potential, and (b) better estimates of the orbits of the dwarf systems. The Sgr Stream is a powerful tool to address the first point, though there is still disagreement about the basic structure of the Galactic Halo from this system (Johnston et al. 2005; Helmi 2004). Ground-based and HST measurements of the space motions of dSph systems have begun to reveal their orbital parameters, but there is at present only modest agreement between individual measurements (e.g. Piatek et al. 2002, 2003; Dinescu et al. 2004). Nonetheless, continued observations with existing techniques as well as space-based interferometric proper motions (SIM and GAIA) of these galaxies are sure to reveal their orbital parameters in the relatively near future.

The certain existence of tides should make it clear that the concept of a 'tidal radius' is at best ill-defined. From an observational standpoint, this is typically measured from the photometric profiles and fits to King or King-like models. The extreme sensitivity of any tidal radius, or, by implication of 'tidal breaks', to the background should now be very clear (consider the interesting history of Fornax: Hodge and Smith 1974; Eskridge 1988a,b; Irwin and Hatzidimitriou 1995; Walcher et al. 2003; Coleman and Da Costa 2005). Moreover, it should be clear that a true King tidal radius is a fiction for any galaxy in which the stars interact internally at any level, and which are bound by a tidal field. This suggests, at the very least, that the utility of spherically symmetric, isotropic dynamical models may be nearing its end (Wilkenson et al. 2002; Kleyna et al. 2001, 2002 explore anisotropic models in some detail). In this respect, tides are likely to elongate systems and cause the velocity distribution to become more radial near the tidal limits of the systems (Piatek and Pryor 1995; Oh et al. 1995).

A possible corollary of this discussion is that tides may dominate the kinematics of dSph systems in particular. So much so, in fact, that dark matter may not be required at all to explain their structures or kinematic properties (Klessen and Zhao 2002; Fleck and Kuhn 2003). This interpretation seems far too strong to me. Specific predictions of large line-of-sight extensions in individual systems are not borne out by observations (Klessen and Zhao 2002; Klessen et al. 2003). Indeed, what's more surprising perhaps is how subtle tidal effects seem to be - that is, until systems are fully disrupted! Tides do not appear to eradicate the need for dark matter halos in dwarf systems, but it is clear they certainly do complicate the kinematic models we require to fully understand these halos. More detailed models that investigate the kinematic behavior of realistic systems in realistic tidal fields would be most helpful.

\section{Nagging Problems}

The ubiquity of DM halos inferred from the kinematics of dwarf systems (tides notwithstanding) poses an interesting dilemma: namely, if systems can be essentially dominated by DM, why must DM halos - especially the smallest ones - have any baryons associated with them? This is simply a restatement of the long-standing 'missing halo' problem of CDM models. For the Milky Way, this implies that each visible dSph system is simply the visible tip of a DM iceberg consisting of dozens of other dark halos that lack baryons (see also Kormendy and Freeman 2004 for a different angle on this problem). As with many 
aspects of CDM, what you do not see is supposed to not bother you. But, for me, it does. If small DM halos are so common, do they ever interact with their visible brethren? If so, what do such interactions do to the kinematics of the systems we can directly study? Why do visible dSph systems outside of 50-70 kpc from the Galaxy center appear to be reasonably undisturbed, while inside this radius we see only streams? If there is truly a disconnect between DM and baryons, shouldn't we see a few very massive - and tidally stable - halos with just enough baryons to be detectable located close to the Milky Way?

The subtlety of substructure seems also to be a problem. Why do so many dSph and $\mathrm{dE}$ galaxies appear to be well-fit by King profiles (or, if you prefer, exponential or Sersic profiles)? These are the baryons, after all; if the DM halos are much more extended so that the cores - where the baryons supposedly reside - are essentially near-constant density harmonic potentials, substructures should be long-lived (Klypin et al. 2003). One might expect the baryons to be distributed in quite chaotic ways within these harmonic cores. Perhaps tidal effects such as those described Mayer et al. 2001 have forced the baryons into a much more restricted family of structural models near the centers of the halo potentials. From the perspective of the DM cores, the homogeneity of the dispersion profiles (Figure 1) raises the inverse question: do the baryons affect the dynamics at all? Also, why are the DM halos all so similar in central density and extent (again, as implied by Figure 1)? CDM models predict a range in these properties, but the DM halos of local dwarfs do not seem to exhibit this range.

These sorts of questions have made dwarf systems a testing ground for non-standard formulations of gravity. MoND (Modified Newtonian Dynamics, Milgrom 1983a,b; Bekenstein 2004) represents the most common form of altered gravity applied to astronomical kinematics. The fact is, MoND fits $\mathrm{dSph}$ and $\mathrm{dE}$ kinematics just as adequately as standard gravity (e.g. Lokas 2001, 2002). But some of the issues raised above, notably tides and the non-spherical structures and anisotropies that tides induce, remain free parameters that allow both CDM and MoND models to fit these data reasonably well. The hope is that as the datasets increase in size and as the models become more refined, a critical test to use dwarf galaxy kinematics to distinguish MoND and DM models may emerge. To this end, it seems to me that it would be interesting to attempt fully-MoNDian n-body simulations of individual dwarf systems in a (MoNDian, naturally) Milky Way potential. The lack of apparent precession in the orbit of the Sgr Stream (Helmi 2004; Johnston et al. 2005) has been cited as evidence against a severely oblate potential (as a disk-like system with no DM must be), but detailed models may provide some surprises.

\section{Postscript from Les Diablerets}

The issues above reflect the contents of my talk at Les Diablerets. Here are some related thoughts - in highly abbreviated form - that developed as I spoke with and listened to various colleagues at the conference:

- Though the kinematic study of both dSph and dE galaxies is now entering a new observational era, we must remain wary of the same problems that people worried about early in this game: precision, binaries and atmospheric jitter. The multiple groups are attacking the problem (for both $\mathrm{dSph}$ and dE galaxies) must carry out critical comparisons of their kinematic datasets; for this to work, all groups need to publish their data as fully and quickly as practical.

- For the dE galaxies, longslit spectra are still the norm (however, see Geha 2004); Two-dimensional mapping of the kinematics of these galaxies will require clever use of integral field units and Fabry-Perot instruments. 
- It is impressive to see how theory has reached the scale of true dwarf systems, but we may still require higher resolution to mimic realistic observational datasets for comparison. The models of Mayer et al. (2001; also at this meeting) provide a solid foundation on which to build such analyses. A definitive guide to the observational signatures of tides would be particularly helpful.

- Substructure is likely to be a very important and useful feature of both dSph and $\mathrm{dE}$ galaxies both as a dynamical probe and a diagnostic feature of the past evolution of individual systems. In this regard, the very interesting result of Tolstoy et al. (2004) illustrates both points beautifully.

- Do dSph and dE galaxies 'kill' MoND? If not, what observations do we need to critically test this idea? There are few systems more fully embedded in the MoND regime and so these dwarfs are likely to play an important role in MoND's success or failure.

- Nature is likely not unimodal with regard to tides vs. DM; I think we should all probably calm down a bit on this issue. Dwarf galaxies near giant galaxies will be affected by tides. On the other hand, dwarf galaxies with massive dark halos will also dictate their own kinematics significantly. Can we disentangle these effects?

Our musings at Les Diablerets has made it clear how rich the Universe of dwarf galaxies is. The primal connection of dwarfs with larger galaxies is well established, independently of which detailed formation scenario one adopts. We will continue to learn more about galaxies in general by understanding dwarf systems in better detail. Given the pace of advances since the last similar meeting - Provence, 1993 - let's plan to do this again, but a bit sooner than in twelve years!

\section{Acknowledgements}

I am happy to thank Matt Walker and Ed Olszewski for their collaboration on our work to study the kinematics of nearby dSph galaxies. I wish to also thank Helmut Jerjen and Bruno Binggeli for the great effort they put into making this such a pleasant and scientifically rewarding meeting. Finally, congratulations and thanks to all at Les Diablerets for their excellent presentations and posters, and for a number of stimulating discussions. This work has been funded in part by grants to MM and EO from the National Science Foundation of the United States.

\section{References}

Aaronson, M. 1983, ApJ 266, L11

Bekenstein, J.D. 2004, Phys Rev D, 70, 083509

Butler, D.J. \& Martínez-Delgado, D. 2005, AJ 129, 2217

Coleman, M.G. \& Da Costa, G.S. 2005, PASA 22, 162

Coleman, M., Da Costa, G.S., Bland-Hawthorn, J., Martínez-Delgado, D., Freeman, K.C. \& Malin, D. 2004, $A J 127,832$

De Rijcke, S., Dejonghe, H., Zeilinger, W.W. \& Hau, G.K.T. 2001, ApJ 559, L21

De Rijcke, S., Dejonghe, H., Zeilinger, W.W. \& Hau, G.K.T. 2004, A $\& A 426,53$

Dinescu, D.I., Keeney, B.A., Majewski, S.R. \& Girard, T.M. 2004, AJ 128, 687

Eskridge, P.B. 1988a, AJ 96, 1352

Eskridge, P.B. 1988b, AJ 96, 1614

Ferguson, A.M.N., Irwin, M.J., Ibata, R.A., Lewis, G.F. \& Tanvir, N.R. 2002, AJ 124, 1452

Fleck, J. \& Kuhn, J.R. 2003, ApJ 592, 147

Geha, M. 2004, American Astronomical Society Meeting Abstracts 205, 169.04

Geha, M., Guhathakurta, P. \& van der Marel, R.P. 2002, AJ 124, 3073 
Geha, M., Guhathakurta, P. \& van der Marel, R.P. 2003, AJ 126, 1794

Gerhard, O.E. \& Spergel, D.N. 1992, ApJ 397, 38

Gómez-Flechoso, M.Á. \& Martínez-Delgado, D. 2003, ApJ 586, L123

Harbeck, D., et al. 2001, AJ 122, 3092

Helmi, A. 2004, ApJ 610, L97

Hodge, P.W. \& Smith, D.W. 1974, ApJ 188, 19

Ibata, R.A., Gilmore, G. \& Irwin, M.J. 1994, Nature 370, 194

Irwin, M. \& Hatzidimitriou, D. 1995, MNRAS 277, 1354

Johnston, K.V., Law, D.R. \& Majewski, S.R. 2005, ApJ 619, 800

Klessen, R.S., Grebel, E.K. \& Harbeck, D. 2003, ApJ 589, 798

Klessen, R.S. \& Zhao, H. 2002, ApJ 566, 838

Kleyna, J.T., Wilkinson, M.I., Evans, N.W. \& Gilmore, G. 2001, ApJ 563, L115

Kleyna, J.T., Wilkinson, M.I., Evans, N.W. \& Gilmore, G. 2004, MNRAS 354, L66

Kleyna, J., Wilkinson, M.I., Evans, N.W., Gilmore, G. \& Frayn, C. 2002, MNRAS 330, 792

Kleyna, J.T., Wilkinson, M.I., Gilmore, G. \& Evans, N.W. 2003, ApJ 588, L21

Kormendy, J. \& Freeman, K. C. in Dark Matter in Galaxies; IAU Symposium 220, p. 377

Łokas, E.L. 2001, MNRAS 327, L21

Lokas, E.L. 2002, MNRAS 333, 697

Martínez-Delgado, D., Alonso-García, J., Aparicio, A. \& Gómez-Flechoso, M.A. 2001, ApJ 549, L63

Mateo, M.L. 1998, ARAA 36, 435

Mayer, L., Governato, F., Colpi, M., Moore, B., Quinn, T., Wadsley, J., Stadel, J. \& Lake, G. 2001a, ApJ 547, L123

Mayer, L., Governato, F., Colpi, M., Moore, B., Quinn, T., Wadsley, J., Stadel, J. \& Lake, G. 2001b, ApJ 559, 754

McConnachie, A.W., Irwin, M.J., Lewis, G.F., Ibata, R.A., Chapman, S.C., Ferguson, A.M.N. \& Tanvir, N.R. 2004, MNRAS 351, L94

Milgrom, M. 1983a, ApJ 270, 365

Milgrom, M. 1983b, ApJ 270, 371

Navarro, J.F., Frenk, C.S. \& White, S.D.M. 1997, ApJ 490, 493

Oh, K.S., Lin, D.N.C. \& Aarseth, S.J. 1995, ApJ 442, 142

Olszewski, E. W., Harris, J., Mateo, M. \& Walker, M. 2005, in preparation

Palma, C., Majewski, S.R., Siegel, M.H., Patterson, R.J., Ostheimer, J.C. \& Link, R. 2003, AJ 125,1352

Piatek, S. \& Pryor, C. 1995, AJ 109, 1071

Piatek, S., et al. 2002, AJ 124, 3198

Piatek, S., Pryor, C., Olszewski, E.W., Harris, H.C., Mateo, M., Minniti, D. \& Tinney, C.G. 2003, AJ 126, 2346

Press, W.H. \& Schechter, P. 1974, ApJ 187, 425

Pryor, C. \& Kormendy, J. 1990, AJ 100, 127

Rojas, B., Miller, B., Lotz, J. \& Hilker, M. 2004, American Astronomical Society Meeting Abstracts 205, 93.05

Shang, Z., et al. 1998, ApJ 504, L23

Tolstoy, E., et al. 2004, ApJ 617, L119

van Zee, L., Skillman, E.D. \& Haynes, M.P. 2004, AJ 128, 121

Walcher, C.J., Fried, J.W., Burkert, A. \& Klessen, R.S. 2003, A\&\&A 406, 847

Walker, M., Mateo, M., Olszewski, E., Bernstein, R., Wang. X. \& Woodroofe, M. 2005, AJ submitted

Wang, X., Woodroofe, M., Walker, M. Mateo, M. \& Olszewski, E. 2005, ApJ June 20 issue

Wilkinson, M.I., Kleyna, J., Evans, N.W. \& Gilmore, G. 2002, MNRAS 330, 778

Wilkinson, M.I., Kleyna, J.T., Evans, N.W., Gilmore, G.F., Irwin, M.J. \& Grebel, E.K. 2004, ApJ 611, L21 